\title{
Radial Distribution Function Imaging by Diffraction Scanning Electron Microscopy
}

\author{
Xiaoke $\mathrm{Mu}^{1,2}$, Di Wang ${ }^{1,3}$, Tao Feng ${ }^{4}$ and Christian Kübel ${ }^{1,2,3}$ \\ 1. Institut für Nanotechnology (INT), Karlsruhe Institute of Technology (KIT), 76344 \\ Eggenstein-Leopoldshafen, Germany \\ 2. Helmholtz-Institute Ulm for Electrochemical Energy Storage (HIU), Karlsruhe Institute of \\ Technology (KIT), 89081 Ulm, Germany \\ 3. Karlsruhe Nano Micro Facility (KNMF), Karlsruhe Institute of Technology (KIT), 76344 \\ Eggenstein-Leopoldshafen, Germany \\ 4. Herbert Gleiter Institute of Nanoscience, Nanjing University of Science and Technology \\ (NJUST), Nanjing, China
}

Interpreting atomic structure of amorphous materials have attracted attentions for a century and, in recent years, especially heterogeneous nanoglasses have fueled the interest because of their unusual structure and properties [1]. However, only few experimental means offer a way to characterize the disordered structures. Atomic radial distribution function (RDF) is one of the important tools for the goal, which was first obtained from X-ray diffraction data of organic solids [2], and afterward extended to electron diffraction for metallic glasses [3]. RDF describes the probability to find certain atomic pairs as a function of the pair separation and consequently, provides structural information in the short and medium range. However, the traditional diffraction experiments only average large sample areas and lack spatial resolution especially at nanometer scale. Plenty information is hidden in the averaged signal.

In this work, we demonstrate a newly developed method, RDF-imaging, combining diffraction in scanning transmission electron microscopy (STEM) mode with RDF analysis and spectral-imaging analysis (e.g. multiple linear least square (MLLS) fitting) to achieve structural mapping of heterogeneous amorphous materials with $1 \mathrm{~nm}$ resolution. Figure 1 schematically shows the procedure for data acquisition and RDF calculation: a 4-dimensional (D) diffraction-image is acquired by recording diffraction patterns in STEM mode with quasi parallel nano-beam configuration (0.8 mrad convergence angle) and $1 \mathrm{~nm}$ spot size. Structure factors are calculated from the diffraction patterns by subtraction and normalization with single atomic scattering factors. The structure factors are then Fourier transformed into RDFs. A 3D data cube of RDFs (RDF-cube) is constructed by relating pixel positions of the initial diffraction-image to the calculated RDFs. The RDF-cube can then be analyzed by MLLS fitting of reference spectra taking experimentally from pure phase or numerically from matrix decomposition by multivariate statistical analysis.

The method is extremely sensitive to atomic packing variation. Figure 2 shows an application to amorphous $\mathrm{ZrO}_{2}\left(\mathrm{a}-\mathrm{ZrO}_{2}\right)$ and a-ZrFe multilayers. Not surprisingly, both the $\mathrm{a}-\mathrm{ZrO}_{2}$ and the a- $\mathrm{ZrFe}$ phases are unambiguously distinguished by RDF-image (figure 2d,e), but also an interface layer between $\mathrm{ZrO}_{2}$ and $\mathrm{ZrFe}$ (figure 2c) is detected, which could not be identified in STEM-EELS and EDX maps. The atomic structure (figure $2 \mathrm{a}$, red dashed-line) of the interfacial layers possesses the same atomic packing as that of the a- $\mathrm{ZrO}_{2}$ phase (figure $2 \mathrm{a}$, blue line) but with a $0.04 \AA$ shrinkage of the average bonding distance. The shift in bonding distance could be introduced by Fe replacing $\mathrm{Zr}$ atoms in the $\mathrm{ZrO}_{2}$ clusters due to diffusion of $\mathrm{Fe}$ atoms from the a- $\mathrm{ZrFe}$ into the $\mathrm{ZrO}_{2}$ layers. 
[1] R Witte, T Feng, JX Fang, A Fischer, M Ghafari, R Kruk, R Brand, D Wang, H Hahn, H Gleiter, Appl Phys Lett 103 (2013), p. 073106.

[2] T Egami and S J L Billinge in "Underneath the Bragg peaks structural analysis of complex materials", (Elsevier Ltd, Kidlington, Oxford, UK), p. 55.

[3] D J H Cockayne and D R Mckenzie, Acta Crystallographica Section A 44 (1988), p. 870.

[4] Authors thank financial support from KNMF and Hi-C project.

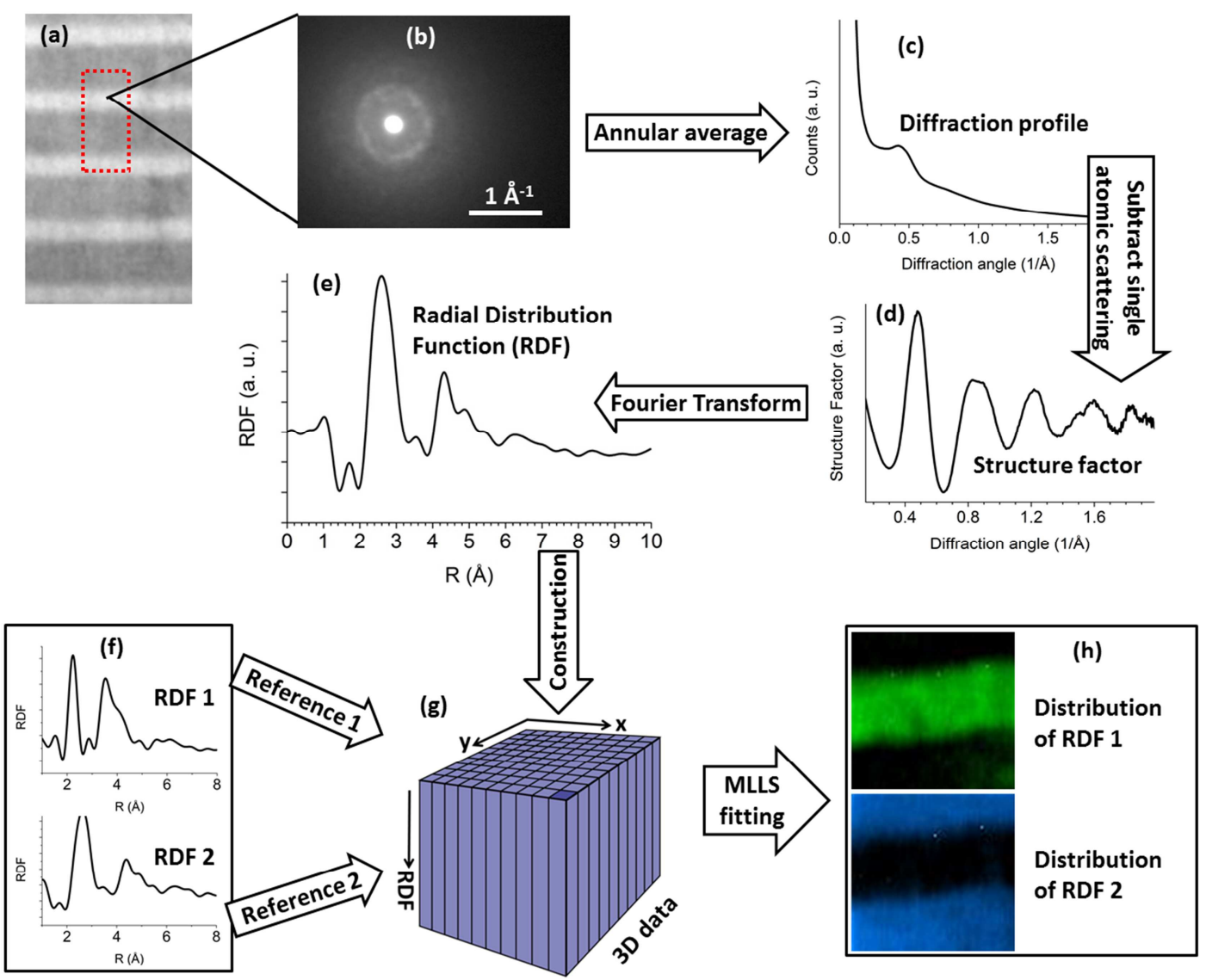

Figure 1. Sketch of procedures to calculate the RDF-cube from experimental STEMdiffraction data and MLLS analysis of the RDF-cube. (a) HAADF image. (b) A typical diffraction pattern in STEM-diffraction. (c) Annular averaged diffraction profile. (d) Structure factor . (e) RDF obtained by Fourier transform of structure factor. (f) Reference RDFs. (g) Sketch of the constructed RDF data cube (RDF-Cube). (h) Imaging results: structural maps.
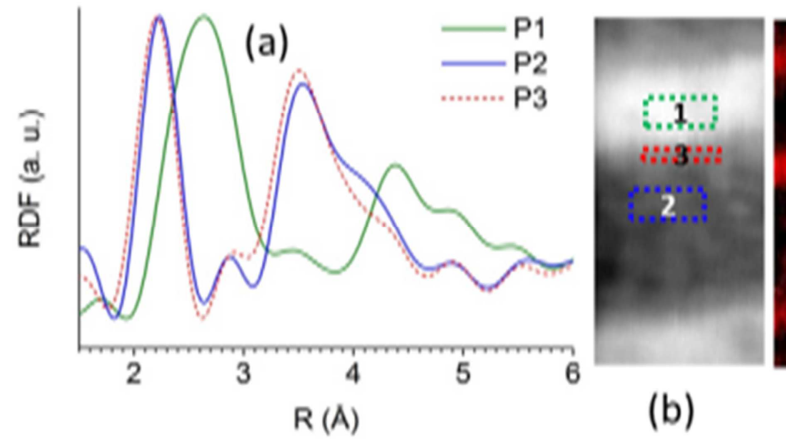

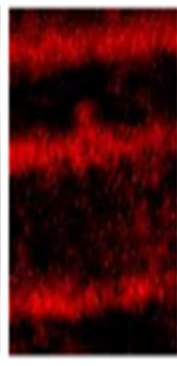

(c)

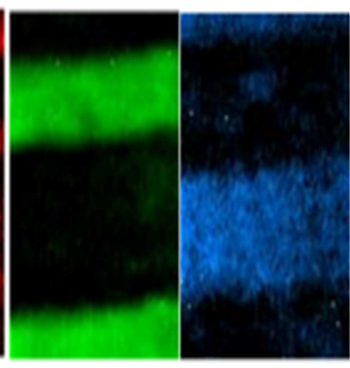

(d) (e)

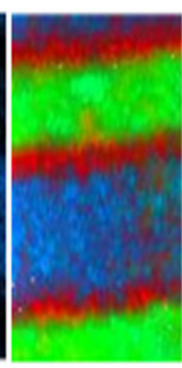

(f)

Figure 2. Results from $\mathrm{ZrO} 2 \mathrm{ZrFe}$ multilayers: (a) RDFs abstracted from the areas indicated by boxes 1, 2 and 3 in (b). (b) STEM-HAADF image. (c) to (d) are maps of the structures according to the RDFs of P3, P1 and P2 in (a). (f) RGB color mix of (c), (d) and (e) 